\title{
Anforderungen an den Facharztstandard bei fachgebietsfremder Tätigkeit
}

\section{Einleitung}

In Deutschland besteht für Patienten grundsätzlich das Recht auf freie Arztwahl. Dies führt nicht nur unter Ärzten derselben Fachgruppe, sondern auch fachgebietsübergreifend zu Konfliktsituationen. Wendet sich ein Patient aufgrund von Beschwerden, die bildgebende Diagnostik erforderlich machen, an einen Nicht-Radiologen bzw. einen sogenannten „Teilradiologen“, so ist dies aus Sicht des Radiologen, dessen Kernleistung die Erbringung dieser bildgebenden Diagnostik bildet, durchaus ein berufspolitisch relevantes Thema.

Zu den „Teilradiologen“ zählen verschiedene Facharztgruppen, darunter Orthopäden, Chirurgen, Angiologen, Kardiologen, Urologen oder Internisten. Sie erwerben im Rahmen Ihrer Facharztausbildung die sogenannte Fach- und Sachkunde, um Röntgenstrahlung am Menschen anwenden und infolgedessen konventionelle Röntgendiagnostik, durchführen zu dürfen. Im Gegensatz zu den Fachärzten für Radiologie haben Teilradiologen jedoch die in mehrfacher Hinsicht problematische Möglichkeit, sich Patienten, nach Stellung einer durch bildgebende Verfahren zu überprüfenden Verdachtsdiagnose, selbst zur bildgebenden Diagnostik zuzuweisen und diese mit eigenen Röntgengeräten durchzuführen.

Welche Facharztgruppe welche Leistungen nach erfolgter Weiterbildung erbringen darf, ist in der (Muster-)Weiterbildungsordnung der Bundesärztekammer sowie in den Weiterbildungsordnungen der Landesärztekammern geregelt. Letztere sind für alle Angelegenheiten ärztlicher Weiterbildung als Körperschaften des Öffentlichen Rechts zuständig. Die von der Bundesärztekammer erarbeitete (Muster-)Weiterbildungsordnung hat für die Landesärztekammern rein empfehlenden Charakter.

Mit den abrechnungsrechtlichen Modalitäten haben sich sodann die Kassenärztlichen Vereinigungen (KV) sowie die Sozialgerichtsbarkeit auseinanderzusetzen. Überschreitet ein Behandler durch eine Untersuchungsmaßnahme jedoch seine Weiterbildungskompetenz, sind die Zivilgerichte zuständig, um etwaige daraus resultierende Behandlungsfehler aus arzthaftungsrechtlicher Sicht zu überprüfen. Dabei ist von entscheidender Bedeutung, aus welchem Facharztstandard heraus das ärztliche Handeln zu überprüfen und zu bewerten ist.

Der Facharztstandard bestimmt sich grundsätzlich losgelöst von der formellen Ernennung zum Facharzt. Der Begriff beschreibt vielmehr die dem Patienten gegenüber geschuldete Sorgfalt im Rahmen der ärztlichen Behandlung. So kann auch bei einer Behandlung durch einen Nicht-Facharzt der Facharztstandard gewahrt sein, sofern dieser bei der Behandlung durch einen Facharzt angeleitet und überwacht wurde. Dabei muss sich der Facharztstandard gegenüber dem Patienten anhand objektiver Kriterien belegen lassen, hierzu zählen Protokolle über ausreichende Anzahl und Schwierigkeit der von dem Nicht-Facharzt bisher durchgeführten Behandlungen, Fortbildungen und Kontrollen sowie über den individuellen Ausbildungsstand.

Die Nichteinhaltung des Facharztstandards hat große haftungsrechtliche Konsequenzen: das Vorliegen einer grob fehlerhaften Unterschreitung des Standards kann zu einer Umkehr der Beweislast in einem zivilrechtlichen Arzthaftungsprozess führen. Auch bei Vorliegen eines einfachen Befunderhebungsfehlers kann eine Beweislastumkehr eintreten, sofern Diagnose- oder Kontrollbefunde behandlungsfehlerhaft nicht - ggf. verspätet - erhoben bzw. gesichert wurden, die Erhebung mit hinreichender Wahrscheinlichkeit ein reaktionspflichtiges Ergebnis erbracht und das Unterlassen einer Reaktion auf einen derartigen Befund sich als grober Behandlungsfehler - ggf. in Form eines fundamentalen Diagnosefehlers - dargestellt hätte (vgl. BGH, VersR 2011, 1148). Die Beweislastumkehr führt dazu, dass bei Komplikationen der medizinischen Behandlungsmaßnahme vermutet wird, dass diese auf einen unzureichenden Behandlungsstandard zurückzuführen sind. Sodann muss entgegen der sonst geltenden Beweislastverteilung nicht der Patient beweisen, dass die Komplikationen auf ärztli-

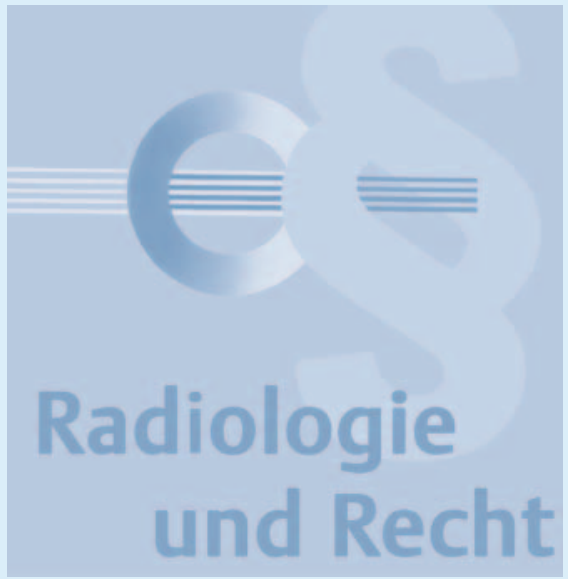

chem Fehlverhalten beruhen, sondern es muss von ärztlicher Seite dargelegt und bewiesen werden, dass auch ein Facharzt der einschlägigen Facharztgruppe den Schaden nicht hätte vermeiden können. Damit begibt sich der jeweilige Behandler in die Defensive.

\section{Rechtsprechung des Bundesverfassungsgerichts zu den Fachgebietsgrenzen}

Die Regelung des Facharztwesens fällt in die ausschließliche Gesetzgebungszuständigkeit der Länder (BVerfG, Beschluss v. 09.05.1972, Az.: 1 BvR 518/62, BVerfGE $33,125,171)$. Die Regelungen zur Einhaltung der Fachgebietsgrenzen sind in den Heilberufsgesetzen bzw. „Kammergesetzen“ der Länder aufgeführt. Die Heilberufsgesetze regeln dabei die Voraussetzungen zur Berufsausübung, die Berufsvertretungen und die Berufsgerichtsbarkeit der Ärzte. Jedes Bundesland besitzt jeweils eigene Heilberufsgesetze.

Gem. § 29 des Hamburgischen Kammergesetzes für die Heilberufe (HmbKGH) können Kammermitglieder „nach Maßgabe dieses Abschnitts neben ihrer Berufsbezeichnung weitere Bezeichnungen führen, die auf besondere Kenntnisse in einem bestimmten beruflichen Gebiet (Gebietsbezeichnung; Facharztbezeichnung im Gebiet), Teilgebiet (Teilgebietsbezeichnung) oder auf andere zusätzlich erworbene Kenntnisse und Fähigkeiten (Zusatzbezeichnung, Zusatzweiterbildung) hinweisen." Grundlage für den Erwerb einer solchen Gebietsbezeichnung oder Facharztbezeichnung ist gem. §35 HmbKGH die Weiterbildungsordnung, eine von der jeweiligen Ärztekammer erlassene 
Satzung. Diese bildet Rahmen und Grenzen der jeweiligen Facharztkompetenzen.

Bereits im Jahr 1972 hat das Bundesverfassungsgericht (BVerfG) entschieden, dass im Bereich des Facharztwesens zumindest die „statusbildenden“ Formen, d. h. diejenigen Regeln, die die Voraussetzungen der Facharztanerkennung, die zugelassenen Facharztrichtungen, die Mindestdauer der Ausbildung, das Verfahren der Anerkennung, die Gründe für eine Zurücknahme der Anerkennung sowie die allgemeine Stellung der Fachärzte innerhalb des gesamten Gesundheitswesens betreffen, in den Grundzügen durch förmliches Gesetz festgelegt werden müssen. Hieraus folgt, dass das Facharztwesen nicht ausschließlich der Regelung durch Satzung der Ärztekammern (Facharztordnungen) überlassen werden darf.

Jedoch haben die Länder das Facharztwesen nicht selbst unmittelbar durch Gesetze ausgestaltet, sondern in den Kammergesetzen den Landesärztekammern das Recht verliehen, die Berufspflichten der Ärzte in einer Berufsordnung zu regeln. Auf Grund dieser Ermächtigungen wurden von den Ärztekammern Berufsordnungen erlassen, welche einen Teil A bezüglich allgemeiner Berufsvorschriften und Teil B bezüglich der Facharztordnung i. e. S. enthalten. Als Satzungen autonomer Berufsverbände sind die Berufsordnungen unmittelbar geltendes Recht. Sie enthalten die materiellen Regeln für die gesamte berufliche Betätigung der Fachärzte, nämlich Vorschriften über die zugelassenen Facharztbezeichnungen, über die Voraussetzungen für die Anerkennung als Facharzt, insbesondere die Dauer der Ausbildung, über das Anerkennungsverfahren, über die Zurücknahme der Anerkennung sowie über die Pflichten der Fachärzte während der Berufsausübung.

Das von den Kammern gesetzte Recht muss dabei seinem materiellen Inhalt nach zwingend mit höherrangigem Recht, vor allem mit dem Grundgesetz (GG), vollständig vereinbar sein, demzufolge beispielsweise die Berufsfreiheit gem. Art. 12 Abs. 1 GG nicht über Gebühr einschränken. Für die Beachtung dieser Schranke der Satzungsautonomie sorgen die - als Rechtsaufsicht ausgestaltete - Staatsaufsicht sowie die Rechtsweggarantie des Art. 19 Abs. 4 GG.
Das BVerfG hat sich in seinem Urteil vom 01.02.2011 (Az.: 1 BvR 2383/10) mit der Verfassungsbeschwerde eines Facharztes beschäftigt, welcher wegen des Tätigwerdens außerhalb seines Fachgebietes berufsgerichtlich verurteilt worden war. Der Beschwerdeführer, ein Facharzt für MundKiefer-Gesichtschirurgie, sah sich wegen der Ausführung von sog. Schönheitsoperationen im Brust-, Bauch- und Oberarmbereich berufsrechtlichen Sanktionen ausgesetzt, gegen die er sich - im Ergebnis erfolgreich - zur Wehr gesetzt hat. Grundsätzlich gilt für Fachärzte das Verbot der Betätigung außerhalb des eigenen Fachgebiets als allgemeine Richtlinie und nicht als eine auch einzelne Ausnahmefälle ausschließende Regelung (vgl. BVerfGE 33, 125, 168; BVerfG, Dreierausschussbeschluss v. 09.01.1984, Az.: 1 BvR 1219/83). Eine Ausnahme kann gelten, wenn eine Überweisung des Patienten zu einem anderen Facharzt für den Patienten unzumutbar wäre, beispielsweise aufgrund eines besonderen Vertrauensverhältnisses zu dem Facharzt oder besonderem Wunsch nach einer Behandlung durch diesen, wie bereits der Hamburgische Berufsgerichtshof für die Heilberufe in seinem durch das BVerfG aufgehobenen Urteil festgestellt hatte (Urteil v. 30.06.2010, Az.: 6 Bf 60/10.HBG).

Die vorangegangenen Entscheidungen des Berufsgerichts und des Berufsgerichtshofs verletzten den Beschwerdeführer nach Auffassung des BVerfG in seiner durch Art. 12 Abs. 1 GG geschützten Berufsfreiheit. Die Gerichte hatten die auf den Bauch-, Brust- und Oberarmbereich bezogene Operationstätigkeit des Beschwerdeführers als eindeutig fachfremd eingestuft. Die weitere Annahme der Berufsgerichte, der Beschwerdeführer verstoße unabhängig vom Umfang seiner gebietsfremden Tätigkeit gegen das durch §31 Abs. $3 \mathrm{HmbKGH}$ aufgestellte Gebot, wonach derjenige, der eine Gebietsbezeichnung führt, grundsätzlich nur in dem geführten Gebiet tätig werden darf, sofern er nur „systematisch“ gebietsüberschreitend tätig werde, sei jedoch mit dem Grundsatz der Verhältnismäßigkeit unvereinbar. Denn das Verbot der Betätigung außerhalb des eigenen Fachgebiets tätig zu werden, werde den verfassungsrechtlichen Anforderungen nur gerecht, wenn es lediglich als allgemeine Richtlinie, die Ausnahmen vorsehe, gelte, und keine zu enge Auslegung stattfinde, da es die Berufstätigkeit des Arztes empfindlich einschränke (vgl. BVerfGE 33, 125, 168).

Die von den Berufsgerichten gewählte Auslegung des $\S 31$ Abs. $3 \mathrm{HmbKGH}$, bei der der Umfang des fachfremden Tätigwerdens des Arztes nicht berücksichtigt werde, sei zwar zur Erreichung des Zwecks, die besonderen Kenntnisse und Fähigkeiten eines Facharztes auf seinem Gebiet zu erhalten, geeignet. Denn ein Facharzt, der nur auf seinem Fachgebiet tätig ist, hat durch diese spezialisierte Tätigkeit in besonderem Maße die Möglichkeit, seine fachärztlichen Fähigkeiten durch ständige Übung weiter zu schulen und seine Fachkenntnisse zu aktualisieren. Nach Auffassung des BVerfG sei eine solche enge Deutung der Norm aber nicht erforderlich, um den durch seine Facharztausbildung erreichten Leistungsstandard eines Facharztes dauerhaft zu gewährleisten. Denn es sei nicht nachvollziehbar, warum sich die Fähigkeiten und Kenntnisse auf dem Gebiet der fachärztlichen Tätigkeit auch durch eine fachfremde Tätigkeit, die in einem nur sehr geringen Umfang ausgeübt werde, verschlechtern sollten. Der Patientenschutz erfordere es nicht, einem bestimmten Fachgebiet zugeordnete Behandlungen nur durch Ärzte dieses Fachgebiets durchführen zu lassen. Die Qualität ärztlicher Tätigkeit werde bereits durch die Approbation nach den Vorschriften der Bundesärzteordnung sichergestellt. Zwar habe ein Arzt im Einzelfall zu prüfen, ob er aufgrund seiner Fähigkeiten und der sonstigen Umstände - wie etwa der Praxisausstattung - in der Lage sei, seinen Patienten nach den Regeln der ärztlichen Kunst zu behandeln. Vorbehaltlich dieser Prüfung sei er aber, unabhängig vom Vorhandensein von Spezialisierungen, berechtigt, Patienten auf allen Gebieten, die von seiner Approbation umfasst sind, zu behandeln. Eine generelle Verpflichtung, Patienten mit Erkrankungen auf einem bestimmten Gebiet an einen für dieses Gebiet zuständigen Facharzt zu verweisen sei damit nicht vereinbar.

Dabei kam es in dem zugrundeliegenden Fall aber darauf an, dass der Umfang der fachfremden Tätigkeit des Facharztes mit 2 Prozent sämtlicher jährlich erbrachter Behandlungsmaßnahmen das für einen Verstoß gegen §31 Abs. 3 HmbKGH relevante Maß nach Auffassung des BVerfG nicht über- 
stieg. Sofern der Anteil fachfremder Tätigkeiten unter einem Gesamtvolumen von 5 Prozent liege, bewege er sich noch im "geringfügigen Bereich“ und stelle somit keinen Verstoß gegen die Regelungen des Kammergesetztes dar.

Dieselben Vorgaben gelten auch für Ärzte, die in einer fachgebiets- und versorgungsbereichsübergreifenden Gemeinschaftspraxis (Berufsausübungsgemeinschaft, BAG) tätig sind, und können durch die Vertretung der Partner in einer solchen Gemeinschaftspraxis nicht überspielt werden. Auch Mitglieder einer BAG sind an die Grenzen ihres jeweiligen Fachgebiets und des Versorgungsbereichs gebunden (vgl. BSG, Urteil v. 14.12.2011, Az.: B 6 KA 31/10 R).

\section{Fachgebietsfremdheit für Vertragsärzte}

Im Rahmen der vertragsärztlichen Versorgung erlangen die Grenzen der Fachgebiete, insbesondere für Erbringung und Abrechenbarkeit ärztlicher Leistungen, erneut besondere Bedeutung: Die Beschränkung auf das Fachgebiet, in dem ein Vertragsarzt zur Behandlung gesetzlich Krankenversicherter zugelassen wird, erfolgt hier bereits mit der Zulassung zur vertragsärztlichen Versorgung. Es dürfen sodann nur Leistungen des eigenen Fachgebiets gegenüber dem gesetzlich krankenversicherten Patienten erbracht und gegenüber der KV abgerechnet werden.

Diese sog. Fachgebietsgrenzen wirken sich bei Aufteilung der Leistungen im Einheitlichen Bewertungsmaßstab (EBM), ihre Einhaltung wird von der Abrechnungsabteilung der jeweiligen KV durch Programme überwacht, die fachfremd abgerechnete Leistungsziffern streichen. Diese Korrekturen der Abrechnung werden als „sachlich-rechnerische Richtigstellung“ bezeichnet. Zu berücksichtigen ist dabei, dass zur Beurteilung einer facheigenen Behandlungsmaßnahme sowohl die Symptomatik des Patienten als auch deren Ursache im Bereich des jeweiligen Fachgebiets des Abrechnenden liegen müssen, um eine Fachgebietszugehörigkeit zu belegen. Nur wenn beide Voraussetzungen erfüllt sind, können auch Leistungen aus Kapiteln des EBM, die dem Fachgebiet nicht zugeordnet sind, abgerechnet werden (vgl. BSG, Urteil v. 08.09.2004, Az.: B 6 KA 39/04 zur Ablehnung der Abrechenbarkeit wg. Fachfremdheit).

\section{Haftungsrechtliche Kon- sequenzen bei Überschreitung der Fachgebietsgrenzen}

Mit der Frage, welcher Facharztstandard bei der Erbringung fachgebietsfremder Tätigkeiten und zur Erkennbarkeit einer richtigen Diagnose sowie zur Beurteilung der Notwendigkeit zu erhebender Befunde aus arzthaftungsrechtlicher Sicht zugrunde zu legen ist, hatte sich das Landgericht Flensburg in seinem Urteil vom 02.08.2019, Az.: 3 O 198/15 zu befassen (zur Abgrenzung von Diagnoseirrtum und Befunderhebungsfehler, vgl. Wigge, Feldmeier-Budelmann, in: RöFo 03/2020, S. 283).

In dem diesem Urteil zugrundeliegenden Sachverhalt hatte eine niedergelassene Fachärztin für Innere Medizin und Pneumologie eine Röntgenuntersuchung des Thorax eines Patienten durchgeführt und im Ergebnis ein vorliegendes Bronchialkarzinom verkannt. Der Patient verstarb während des Verfahrens.

Die Beklagte erhob die Anamnese des Patienten und führte eine Röntgenuntersuchung des Thorax durch. Dabei stellte sie fest: „Unauffälliger Befund an Lunge, Herz, Pleura und Mediastinum. Kein Hinweis auf Pneumonie, kein Anhalt für Tumor." sowie „PROC WV wenn Beschwerden länger als 4 Wochen anhaltend, dann Thorax-CT ..... Vier Monate später stellte sich der Patient erneut vor und klagte über persistierende Schmerzen im Burstkorb, woraufhin nach klinischer Untersuchung und Lungenfunktionstest die Diagnose Asthma bronchiale gestellt wurde. Erneut wurde vermerkt: „Wenn in 2 Wochen nicht besser, dann kurzfristig CT-Thorax ... PROC WV 3 m, wenn nicht besser, dann kurzfristig CT-Thorax. “

Vier Wochen später wurde der Patient stationär aufgenommen und im Rahmen einer im Krankenhaus durchgeführten Röntgenuntersuchung ein „... Verplumpter Hilus links sowie Plattenatelektase im linken Mittelfeld“ festgestellt. Der Befund wurde mittels kontrastmittelgestütztem CT überprüft und ein Lungenkarzinom links zentral mit der „Tumorformel: cT4 NO/2 Mx (entspricht Stadium III A/B) ... Adenokarzinom“ diagnostiziert.

Streitig war in dem anschließenden Rechtsstreit, ob der Pneumologin ein Behandlungsfehler vorzuwerfen sei, wobei es letztlich entscheidend auf die Einordnung des zugrunde zu legenden Facharztstandards hinsichtlich der Durchführung der ThoraxRöntgenaufnahme ankam.

Das Landgericht entschied, sachverständig beraten, dass die Durchführung und Befundung einer Röntgenaufnahme des Thorax zumindest auch (noch) in das Fachgebiet eines niedergelassenen Pneumologen gehöre. Bei der Durchführung und anschließenden Befundung einer Röntgenaufnahme des Thorax habe ein niedergelassener Facharzt für Innere Medizin und Pneumologie deshalb den Standard seines Fachgebiets und nicht den eines Radiologen zu wahren.

Ein Behandlungsfehler liegt nicht erst vor, wenn eine Maßnahme „zwingend“ geboten war, sondern bereits dann, wenn ihr Unterbleiben dem im Zeitpunkt der Behandlung bestehenden medizinischen Standard zuwiderlief (vgl. BGH Urteil v. 22.02.2015, Az.: VI ZR 67/15). Mit dem fachlichen Standard ist derjenige Standard gemeint, der von Angehörigen des jeweiligen Fachs bei Durchführung der jeweiligen Behandlung einzuhalten ist, also eine Behandlung, wie sie ein durchschnittlich qualifizierter, gewissenhafter und aufmerksamer Behandler des jeweiligen Fachgebietes nach dem jeweiligen Stand von medizinischer Wissenschaft und Praxis an Kenntnissen, Wissen, Können und Aufmerksamkeit zu erbringen in der Lage ist (vgl. Martis/Winkhart, Arzthaftungsrecht Fallgruppenkommentar, 5. Aufl. 2018, S. 1135 Rn. P13).

Eine Haftung der erstbehandelnden Pneumologin lehnte die Kammer vorliegend jedoch ab, da ein Diagnosefehler nicht vorliege. Zwar zeigte sich bei der ex post durchgeführten Nachbefundung des ersten Röntgenbildes durch den im Verfahren bestellten Sachverständigen eine links-hiläre tumoröse Raumforderung. Diese hatte die Pneumologin bei der Auswertung des Röntgenbildes verkannt. Eine solche Fehlinterpretation erhobener Befunde (sog. „Diagnoseirrtum“) 
werde in der Rechtsprechung aber nur zurückhaltend als Behandlungsfehler (sog. „Diagnosefehler“) bewertet: Ein Behandlungsfehler sei nicht immer schon dann anzunehmen, wenn ein Arzt zu einer objektiv unrichtigen Diagnose gelange. Diagnoseirrtümer, die objektiv auf eine Fehlinterpretation der ordnungsgemäß erhobenen Befunde zurückzuführen seien, könnten aufgrund der mit der Befundinterpretation naturgemäß verbundenen Schwierigkeiten nur mit Zurückhaltung als Behandlungsfehler gewertet werden (vgl. BGH, Urt.v. 08.07.2003, Az.: I ZR 304/02). Dem stehe auch nicht entgegen, dass der von dem Sachverständigen herangezogene Radiologe in seinem schriftlichen Gutachten ausgeführt habe, die beschriebene Raumforderung hätte vermutet und in einem schriftlichen Befund Erwähnung finden müssen. Maßgeblicher ärztlicher Standard für die Beurteilung sei hier der eines Facharztes für Innere Medizin und Pneumologie, nicht derjenige eines Radiologen. Dieser Facharztstandard sei von der Pneumologin durch die gestellte, objektiv fehlerhafte Diagnose nicht in vorwerfbarer Weise unterschritten worden.

Ausgangspunkt für die Einordnung, ob der Facharztstandard unterschritten wurde, sind die Weiterbildungsinhalte der Facharztausbildung. In der hier einschlägigen Weiterbildungsordnung der Ärztekammer SchleswigHolstein in der seit dem 25.05.2011 geltenden Fassung war für das Fachgebiet der „Inneren Medizin“ (Nr. 13.1 WBO) die Befundung einer Röntgenaufnahme nicht ausdrücklich erwähnt, auch nicht unter den dort definierten Untersuchungs- und Behandlungsverfahren. Gleiches galt für das Fachgebiet der „Inneren Medizin und Pneumologie“ (Nr. 13.8 WBO). Demgegenüber gehörte die radiologische Diagnostik an Thorax und Thoraxorganen zu den definierten Untersuchungs- und Behandlungsverfahren des Fachgebiets der „Radiologie“ (Nr. 29 WBO).

Der Sachverständige führte in dem Verfahren jedoch aus, dass die Befundung von Röntgenbildern zwar nicht Bestandteil der Facharztausbildung für Innere Medizin und Pneumologie, nämlich weder Ausbildungsnoch Prüfungsvoraussetzung, eine eigene Anfertigung und Befundung von Röntgenbildern durch einen niedergelassenen internistischen Pneumologen aber üblich sei.
Aus ärztlicher Sicht sei nicht festzustellen, dass ein Pneumologe keine Röntgenbilder befunden dürfe. Es sei aber eine schwierige Diagnostik.

Aufgrund der Einschätzung des Sachverständigen, dass auch Pneumologen grundsätzlich Röntgenbilder anfertigen und befunden dürften, konnte die Kammer nicht feststellen, dass die Durchführung und Befundung einer Röntgenaufnahme allein in das Fachgebiet der Radiologie falle, sondern für pneumologische Sachverhalte aufgrund einer bestehenden Übung auch in das Fachgebiet der Inneren Medizin und Pneumologie. Aus diesem Grund hatte die Beklagte nach Auffassung der Kammer keine Untersuchungs- und Behandlungsmethoden eines fremden Fachgebiets angewandt, sondern (auch) die ihres eigenen. Damit war der ärztliche Standard eines Facharzts für Innere Medizin und Pneumologie für die Beurteilung maßgeblich und die Beklagte hatte keine fachfremde Leistung erbracht, was zu der Einordnung des Verkennens des Bronchialkarzinoms als Behandlungsfehler hätte führen können. Denn die Anforderungen an die Befundung einer Röntgenaufnahme durch einen Radiologen werden sachverständig höher angesetzt, da die Befundung zu deren Kernbereich zählt, anders als bei einem Facharzt für Innere Medizin und Pneumologie.

Das Gebiet der Radiologie umfasst laut (Muster-)Weiterbildungsordnung die Erkennung von Krankheiten mit Hilfe ionisierender Strahlen, kernphysikalischer und sonographischer Verfahren sowie die Anwendung interventioneller, minimal-invasiver radiologischer Verfahren in der Erwachsenen-, Kinder und Neuroradiologie sowie die Belange des Strahlenschutzes. Durch die fünf- bis siebenjährige Facharztweiterbildung in einem Krankenhaus, einem radiologischen oder nuklearmedizinischen Institut wird der Facharztstandard des Radiologen in Bezug auf die diagnostische und therapeutische Radiologie naturgemäß strenger bewertet. Unerheblich ist dabei, ob es sich um einen niedergelassenen oder einen in einem Krankenhaus tätigen Radiologen handelt.

Im Grundsatz gilt, dass ein Patient ausgehend von seinem Erwartungshorizont bei Aufsuchen eines Arztes Anspruch auf Einhaltung des Standards guter Versorgung nach Maßstab eines erfahrenen Arztes der jeweiligen Fachrichtung („Facharztstandard“) hat (Frahm/Walter, Arzthaftungsrecht, 6. Auflage, Rn. 91 m.w.N.). Da es sich bei der Beklagten um eine Fachärztin für Innere Medizin und Pneumologie handelte, wäre dies auch der zugrunde zu legende Facharztstandard.

In einem ähnlich gelagerten Fall, bei dem ein Allgemeinmediziner mit eigener Röntgendiagnostik ebenfalls ein Bronchialkarzinom übersah, hat das Schleswig-Holsteinische Oberlandesgericht dementsprechend den Standard eines Allgemeinarztes zugrunde gelegt (OLG Schleswig, Urteil v. 24.06.2005, Az.: 4 U 10/04).

Bei der Beurteilung eines medizinischen Geschehens hat das Gericht demnach auf die Fachkenntnisse des betroffenen medizinischen Sachgebiets abzustellen. Wendet ein Arzt aber Untersuchungs- und Behandlungsmethoden an, die in ein fremdes Fachgebiet fallen, hat er dessen Standard zu garantieren (LG Flensburg, Urteil v. 28.02.2019, Az.: 30 5/14). In dem diesem Urteil zugrunde liegenden Sachverhalt hatte ein Frauenarzt eine Mammographie fehlgedeutet und ein Mammakarzinom verkannt. Insoweit wurde der anerkannte fachliche Standard guter ärztlicher Versorgung unterschritten. Das Landgericht stellte klar, dass die Durchführung und ausschließliche Befundung einer Mammographie in das Fachgebiet der Radiologie gehöre. Denn die Auswertung von Mammographien sei in der Facharztausbildung zur Frauenheilkunde weder damals noch heute enthalten. Bei der Durchführung und ausschließlichen Befundung einer Mammographie habe deshalb ein Facharzt für Frauenheilkunde und Geburtshilfe den Standard eines Facharztes für Radiologie zu wahren. Dieser Facharztstandard war durch den dortigen Beklagten unterschritten worden, sodass ein vorwerfbarer Diagnosefehler vorlag.

Gehört also die Durchführung und Befundung einer radiologischen Aufnahme allein in das Fachgebiet der Radiologie, ist demnach der maßgebliche Facharztstandard allein der Facharztstandard eines Radiologen. 


\section{Fazit}

Bei der Beurteilung, welcher Facharztstandard für die Bewertung einer ärztlichen Behandlung zugrunde zu legen ist, ist auf den Erwartungshorizont eines durchschnittlichen, mündigen Patienten abzustellen. Die verbindliche Einordnung ärztlichen Handelns erfolgt in Zivilprozessen jedoch durch den jeweiligen gerichtlich bestellten Sachverständigen.

Sofern in verwaltungsrechtlicher Hinsicht der Anteil fachfremder Tätigkeiten unter einem Gesamtvolumen von 5 Prozent liegt, sich damit noch im erlaubten „geringfügigen Bereich“ bewegt und somit keinen Verstoß gegen die Regelungen der Heilberufs- und Kammergesetzte darstellt, ist im Rahmen der inhaltlichen Überprüfung des ärztlichen Handelns auf die Weiterbildungsordnungen abzustellen. Zur Einordnung des ärztlichen Handelns unter eine Facharztgruppe wird dabei seitens der Zivilgerichte die Weiterbildungsordnung der jeweiligen Landesärztekammer in der zum Zeitpunkt des vermeintlich patientenschädigenden Verhaltens ex ante geltenden Fassung herangezogen.

Auf Grundlage der Vorgaben der Weiterbildungsordnung erfolgt sodann die sachverständige Bewertung, welche Sorgfalt von dem beklagten Arzt zur Erfüllung des Facharztstandards hätte erwartet werden können und müssen.

Die vorgenannten Sachverhalte belegen, dass eine unmittelbar gute Diagnostik und Befundung nicht nur unnötige weitere Diagnostik, Operationen oder Behandlungen vermeiden und Krankheitsdauern verkürzen, sondern sogar unmittelbar Leben retten kann. Dies bestätigt die Sicht der Radiologen, dass Teilradiologen die komplexe Aufnahmetechnik nicht in gleichem Maße beherrschen wie Radiologen selbst und demzufolge mit eigenen Geräten möglicherweise weder optimale Bilder zur Diagnostik und Befundung erstellen, noch diese anschließend auf höchstem Standard interpretieren bzw. einordnen können. Diese Gefahr gilt auch und insbesondere hinsichtlich der Magnetresonanztomographie, als im Vergleich zu konventionellen Röntgendiagnostik wesentlich schwieriger zu erlernendes und erheblich komplexeres diagnostisches Verfahren. Diesem Umstand tragen auch Sachverständige in Zivilprozessen Rechnung, indem der Standard der Radiologen in deren Kernbereichen als deutlich verschärft beurteilt wird.

Auch wenn die Teilradiologen häufig einen sich aus der Selbstzuweisung ergebenden zeitlichen Vorteil anführen, muss darauf hingewiesen werden, dass dieser Vorteil nur solange gewahrt bleibt, wie auch die richtige Diagnose gestellt wird, sodass in Folge eine adäquate Therapie eingeleitet werden kann. Zwar kennt ein Teilradiologe im Zweifel die klinischen Zusammenhänge des von ihm persönlich klinisch und bildgebend diagnostisch untersuchten Patienten besser als ein hinzugezogener Radiologe. Sofern jedoch einem Radiologen ein ordnungsgemäß und vollständig ausgefüllter Überweisungsschein übermittelt wird und der überweisende Arzt zusätzlich eine schriftliche oder telefonische Beschreibung der bereits erfolgten klinischen Untersuchungen des Patienten bereitstellt, erfolgt eine ganzheitliche und voll fachlich qualifizierte Befundung unter Berücksichtigung aller medizinischen Faktoren durch den Radiologen im Dienste der Patientensicherheit und Effizienz des Gesundheitssystems. Durch ein geteiltes System von klinischem Behandler und Radiologen werden zudem Anreize zur Selbstüberweisung durch Teilradiologen vermieden. Dies dient wiederum dem, im Sozialrecht normativ verankerten, Wirtschaftlichkeitsgebot.

Im Einzelfall hat somit jeder Facharzt sorgfältig zu prüfen, ob er den Patienten nach dem geltenden Facharztstandard fachgerecht behandeln kann, um sich keinem Haftungsrisiko auszusetzen. Anderenfalls drohen nicht nur berufsrechtliche sowie zivil- und/oder strafrechtliche Konsequenzen, sondern bei Vertragsärzten auch sachlich-rechnerische Berichtigungen der Abrechnungen mit anschließenden Regressforderungen.

Prof. Dr. Peter Wigge

Rechtsanwalt

Fachanwalt für Medizinrecht

Christina Feldmeier-Budelmann

Rechtsanwältin

Fachanwältin für Medizinrecht

Rechtsanwälte Wigge

Großer Burstah 42

20457 Hamburg

Telefon: (040) 3398 705-90

Telefax: (040) 3398 705-99

E-Mail: hamburg@ra-wigge.de www.ra-wigge.de 